\title{
Towards RIP using free-electron laser SFX data
}

\author{
Lorenzo Galli, Sang-Kil Son, Thomas A. White, Robin Santra, Henry N. \\ Chapman and Max H. Nanao
}

J. Synchrotron Rad. (2015). 22, 249-255

\section{IUCr Journals CRYSTALLOGRAPHY JOURNALS ONLINE}

Copyright (C) International Union of Crystallography

Author(s) of this paper may load this reprint on their own web site or institutional repository provided that this cover page is retained. Republication of this article or its storage in electronic databases other than as specified above is not permitted without prior permission in writing from the IUCr.

For further information see http://journals.iucr.org/services/authorrights.html 
Journal of

Synchrotron

Radiation

ISSN 1600-5775

Received 31 August 2014

Accepted 21 December 2014

\section{Towards RIP using free-electron laser SFX data}

\author{
Lorenzo Galli, ${ }^{\mathrm{a}, \mathrm{b}}$ Sang-Kil Son, ${ }^{\mathrm{a}, \mathrm{c}}$ Thomas A. White, ${ }^{\mathrm{a}}$ Robin Santra, ${ }^{\mathrm{a}, \mathrm{c}, \mathrm{d}}$ \\ Henry N. Chapman ${ }^{\mathrm{a}, \mathrm{b}, \mathrm{c}}$ and Max H. Nanao ${ }^{\mathrm{e}}$ *
}

\author{
${ }^{a}$ Center for Free-Electron Laser Science, DESY, Notkestrasse 85, 22607 Hamburg, Germany, \\ ${ }^{\mathbf{b}}$ Department of Physics, University of Hamburg, Luruper Chaussee 149, 22761 Hamburg, \\ Germany, ' $T$ The Hamburg Centre for Ultrafast Imaging, Luruper Chaussee 149, 22761 Hamburg, \\ Germany, dDepartment of Physics, University of Hamburg, Jungiusstrasse 9, 20355 Hamburg, \\ Germany, and ${ }^{\mathbf{e}} \mathrm{EMBL}$, Grenoble Outstation, Rue Jules Horowitz 6, 38042 Grenoble, France. \\ *E-mail: mnanao@embl.fr
}

Here, it is shown that simulated native serial femtosecond crystallography (SFX) cathepsin B data can be phased by rapid ionization of sulfur atoms. Utilizing standard software adopted for radiation-damage-induced phasing (RIP), the effects on both substructure determination and phasing of the number of collected patterns and fluences are explored for experimental conditions already available at current free-electron laser facilities.

\section{Introduction}

The properties of X-ray free-electron lasers (FELs) and associated sample environments have catalyzed the development of new data collection methods, such as serial femtosecond crystallography (SFX). In SFX, a complete dataset can be obtained by exposing thousands of randomly oriented crystals to the X-ray beam (Chapman et al., 2011; Boutet et al., 2012; Redecke et al., 2013; Johansson et al., 2012). One very attractive feature of this method is that crystals of very small sizes are sufficient to obtain high-quality data, allowing data to be collected from smaller crystals. These experiments also offer the exciting possibility of determining structures that are free of the normal types of radiation damage, since the diffracting pulse ends before most of the processes induced by X-ray absorption can occur. To date, SFX crystal structures show no signs of the kinds of radiation damage previously observed at synchrotron sources (Boutet et al., 2012), even though the dose at which the single crystal is exposed can exceed 100 times the dose limit at cryogenic temperature (Owen et al., 2006). At such doses there is enough X-ray fluence to cause ionization of every atom in the sample (Chapman et al., 2011) and the exposure time is about a million times faster than neutralizing electron recapture processes. Despite the lack of secondary radiation damage in SFX data, ionization of atoms in the sample is unavoidable since it occurs within the femtosecond timescale of the pulse (Hau-Riege et al., 2004; Quiney \& Nugent, 2011). This change in the electronic configuration of atoms leads to the modification of the atomic scattering factors during diffraction, especially at photon energies near absorption edges (Son et al., 2011, 2011a). It has been theorized that, for X-ray fluences already achievable with existing FELs, this ionization could significantly alter the scattering factors of the heavy atoms, hindering the direct application of anomalous phasing methods (Son et al., 2011, 2013). This is analogous to the situation in synchrotron macromolecular crystallography, where radiation damage, if unaccounted for, can dramatically hinder anomalous phasing (Ravelli et al., 2005). In the SFX case, ionization introduces the exciting possibility of determining phases de novo by varying the fluence and hence the scattering factors of the heavy atoms. This new approach could represent a powerful method of experimental phasing which would not require the modification of the native protein crystals if the sulfur atoms already present in the structure could be used. Since ionization occurs at all sulfur atoms and not just those that are found in disulfide bonds, this kind of electronic modification could be of broader applicability than synchrotron-radiation-induced phasing (RIP), which requires disulfide bonds and/or metals. To avoid confusion with phasing by synchrotron radiation damage at cryotemperatures (Ravelli et al., 2003; Evans et al., 2003), and to highlight the central role of the X-ray intensity, we call this new technique 'high-intensity radiation-induced phasing' (HI-RIP).

In this paper it is demonstrated that HI-RIP can in theory be used to determine substructures of radiation damage as well as determine high-quality phases. We show this by simulating two SFX experiments, at high and low X-ray fluence, under experimental conditions that mimic those available at the Linac Coherent Light Source (LCLS) (Emma et al., 2010; Boutet \& Williams, 2010). Furthermore, the effect of different experimental parameters such as variable fluences and the number of patterns on the quality of the substructure solution and phasing is explored. 


\section{Models and methods}

To simulate SFX experiments, it is necessary to take into account ionization dynamics and the accompanying changes of atomic scattering factors (Son et al., 2011). In light atoms, after absorbing an X-ray, an inner-shell electron is ionized and the resulting vacancy is relaxed via Auger decay, typically within a few femtoseconds, or fluorescence. A deep inner-shell vacancy in a heavy atom that has more than two subshells undergoes a cascade of Auger decays, ejecting several electrons. At high $\mathrm{X}$-ray intensity, photoionizations may occur sequentially even to the same atom, resulting in highly charged ions. Even though X-ray absorption locally occurs to the single atom, charge states are redistributed to neighboring atoms during or after ionization processes in the femtosecond regime. Electrons ejected from photoionizations or Auger decays induce a cascade of collisional ionization that occurs within about 100 fs. These X-ray ionization dynamics happen stochastically to every single atom in the sample. Once charged states are created, the atomic scattering factors are altered. Assuming that individual atoms are ionized independently, the scattering intensity is evaluated by (see Appendix $A$ for details)

$$
\begin{aligned}
\frac{\mathrm{d} I(\mathbf{Q}, \mathcal{F}, \omega)}{\mathrm{d} \Omega}= & \mathcal{F} \\
& C(\Omega) \sum_{X}\left\{\left|F_{X}^{0}(\mathbf{Q})\right|^{2} \tilde{a}_{X}(\mathbf{Q}, \mathcal{F}, \omega)\right. \\
& \left.+N_{X}\left|f_{X}^{0}(\mathbf{Q})\right|^{2}\left[a_{X}(\mathbf{Q}, \mathcal{F}, \omega)-\tilde{a}_{X}(\mathbf{Q}, \mathcal{F}, \omega)\right]\right\} \\
& +\mathcal{F} C(\Omega) \sum_{X} \sum_{Y>X}\left|F_{X}^{0}(\mathbf{Q})\right|\left|F_{Y}^{0}(\mathbf{Q})\right| \\
& \times\left[B_{X Y}(\mathbf{Q}, \mathcal{F}, \omega) \cos \Delta \varphi_{X Y}^{0}\right. \\
& \left.+C_{X Y}(\mathbf{Q}, \mathcal{F}, \omega) \sin \Delta \varphi_{X Y}^{0}\right]
\end{aligned}
$$

where $\mathbf{Q}$ is the momentum transfer, $\mathcal{F}$ is the $\mathrm{X}$-ray fluence, and $\omega$ is the photon energy. Here, $X$ is one of the atom species in the sample. $F_{X}^{0}(\mathbf{Q})$ is the structure factor for $X$, including neither dispersion correction nor ionization, and $\Delta \varphi_{X Y}^{0}$ is the phase difference between the structure factors for $X$ and $Y$. In contrast to Son et al. (2011a), here the effects of ionization dynamics and anomalous scattering are considered for all atoms in the sample, which are included in the anomalous coefficients. The atom-specific coefficients of $a_{X}$ and $\tilde{a}_{X}$, and the biatom-specific coefficients of $B_{X Y}$ and $C_{X Y}$ are defined in Appendix $A$. Note that these coefficients depend on both the photon energy $\omega$ and the fluence $\mathcal{F}$. Since the dynamical fluctuations of the atomic form factors during the intense X-ray pulse are dramatic (Son et al., 2013), time-averaged form factors are not sufficient to describe the scattering patterns at high X-ray intensity.

The XATOM toolkit (Son \& Santra, 2012) provides a way to simulate the electronic damage dynamics and to compute the anomalous coefficients present in equation (1), for various atomic species at a chosen X-ray fluence and energy. In Fig. 1, the fluence dependence of the scattering strengths (coefficient $\tilde{a}$ ) for the atomic species of a native protein (i.e. containing only $\mathrm{H}, \mathrm{C}, \mathrm{O}, \mathrm{N}$ and $\mathrm{S}$ ) is plotted, in the case of a $6 \mathrm{keV}, 10 \mathrm{fs}$ flat-top pulse. The plot shows that, at low photon fluence, the relative scattering strength is close to unity, which means that the scattering strength is very similar to the 'synchrotron' case

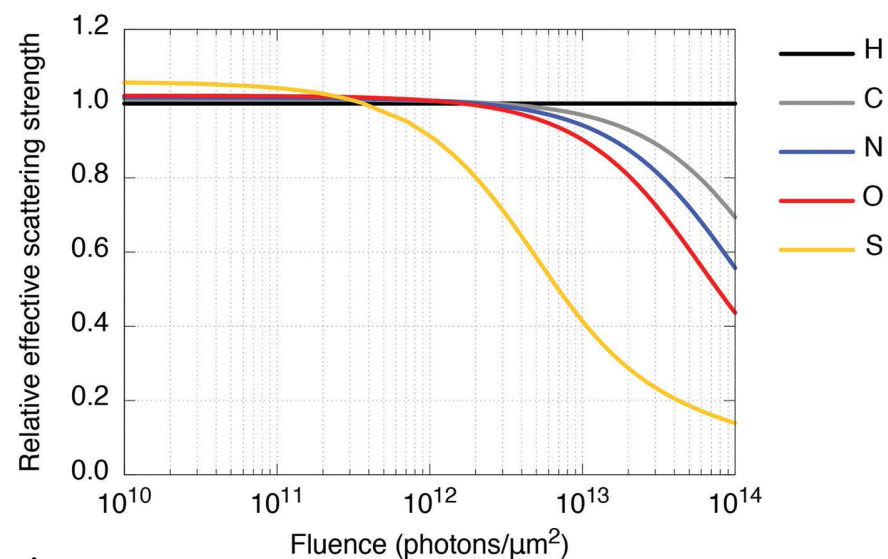

Figure 1

Fluence (photons $/ \mu \mathrm{m}^{2}$ )

Relative effective scattering strength as a function of the X-ray fluence, for different atomic species, at $6 \mathrm{keV}$.

where the ionization is negligible. No dependence of coefficient $\tilde{a}$ on the fluence implies that contribution to the scattering intensity is linearly proportional to the fluence if anomalous coefficients $B$ and $C$ are zero. By contrast, at higher fluence, the scattering strength of $S$ in particular is reduced compared with lighter atoms, owing to its large photoabsorption cross section. Up to about $10^{12}$ photons $\mu \mathrm{m}^{-2}$ for example, the high-intensity modification of the scattering factors of the light atoms is still negligible, while the sulfur has lost almost $40 \%$ of its effective scattering strength.

In principle, it is possible to apply the same strategy as used in the high-intensity multiwavelength anomalous diffraction (MAD) phasing technique (Son et al., 2011). Assuming that all anomalous coefficients are determined theoretically or experimentally, the number of unknowns in equation (1) are $2 N\left[\left|F_{X}^{0}(\mathbf{Q})\right|\right.$ and $\varphi_{X}^{0}(\mathbf{Q})$ for $\left.X=1, \ldots, N\right]$, where $N$ is the number of different atomic species in the sample. To solve these unknowns, a set of $2 N$ linearly independent equations is needed. Since the anomalous coefficients vary not only with wavelength but also with fluences as shown in Fig. 1, one has to measure $2 \mathrm{~N}$ complete datasets at different fluences. In this sense, it should be called a multi-fluence anomalous diffraction method. However, as discussed in detail later on, it might be difficult to accurately measure the molecular structure factors at several distinct fluences using current SFX technology. Instead, one can simply exploit the bleaching effect of the heavy atoms at two fluence points: low fluence for the undamaged case, and high fluence for the damaged case. This bleaching effect can be thought of as being analogous to the specific radiation damage observed at synchrotron sources and, as a result, RIP or SIR (single isomorphous replacement) are the natural phasing methods to use. By performing two SFX experiments at different X-ray fluences, it should therefore be possible to determine the heavy-atom positions and then determine phases.

To retrieve the sulfur coordinates from the change of ionization, two X-ray fluences must be chosen: a 'low dose' fluence and a high fluence (which may be the highest achievable at the beamline) that modifies the scattering properties of sulfurs while leaving light elements relatively 
unchanged. Currently, the coherent X-ray imaging (CXI) endstation at LCLS can provide a SFX setup with a beam that can be focused nominally to $0.2 \mu \mathrm{m}$ of diameter using Kirkpatrick-Baez mirrors, providing up to $0.5 \mathrm{~mJ}$ per pulse in the photon energy range $6-10 \mathrm{keV}$. $6 \mathrm{keV}$ is chosen because the photoabsorption cross section of sulfur is the highest within that energy range, and the maximum fluence per pulse achievable at the focal spot theoretically reaches $10^{13}$ photons $\mu \mathrm{m}^{-2}$. Low fluence pulses can be obtained by attenuating the beam with $\mathrm{Si}$ attenuators. Based on the ionization dynamics calculations, here the amount of damage at $10^{11}$ photons $\mu \mathrm{m}^{-2}$ is judged to be negligible, and this value is set as the lowest intensity.

\section{Simulations}

The Trypanosoma brucei cathepsin B structure recently solved at LCLS [Protein Data Bank (PDB) code 4hwy] (Redecke et $a l$. , 2013), space group $P 4_{2} 2_{1} 2, a=b=125.4 \AA, c=54.56 \AA$, was used to test the method. This protein consists of 340 residues, and includes $19 \mathrm{~S}$ atoms (in 5 methionines and 14 cysteines). The complex-valued structure factors $F_{X}^{0}(\mathbf{Q})$ of equation (1) [and hence their phases $\varphi_{X}^{0}(\mathbf{Q})$ ] were computed separately for each atomic species $X$ (excluding hydrogen) present in the protein structure using the sfall program (Ten Eyck, 1977; Winn, 2011); Friedel mates were averaged using an ad hoc script. The atom- and biatom-specific coefficients were calculated with the XATOM toolkit and the final scattered intensity for each Bragg reflection was calculated using equation (1). In other words, structure factors without any fluence effects were calculated with sfall, and modified to include high-fluence effects with XATOM. The scattering contribution of the bulk solvent region was generated starting from the PDB using the ano_sfall.com script (Holton et al., 2014) and was summed with the scattering factors of the protein.

The SFX experiment was simulated with partial_sim, which is part of the CrystFEL suite (White et al., 2012, 2013). The program takes a list of fully integrated reflection intensities and generates partial reflection intensities of randomly oriented crystals, adding noise to simulate other measurement errors. The detector geometry was chosen to reproduce the 64 tiles of the Cornell-SLAC Pixel Array Detector (CSPAD) (Hart et al., 2012) installed at the CXI endstation. The sampleto-detector distance was set to $11 \mathrm{~cm}$, giving a resolution limit of about $2.7 \AA$ at the corners of the detector for $6 \mathrm{keV}$ energy. The simulated noise and the beam parameters were selected in order to produce $R_{\text {split }}$ (the error metric commonly used for SFX experiments) (White et al., 2012) and $\langle I / \sigma\rangle$ values as close to a real experiment as possible (see, for example, Redecke et al., 2013). Two sets of $3 \times 10^{5}$ patterns were generated, one with $10^{13}$ photons $\mu \mathrm{m}^{-2}$ (as 'high fluence') and another using $10^{11}$ photons $\mu \mathrm{m}^{-2}$ (as 'low fluence'). The simulated noisy partial intensities were then merged using process_hkl (another program from the CrystFEL suite), using a Monte Carlo integration method (Kirian et al., 2010). To test the convergence of the Monte Carlo procedure, smaller subsets of the complete data set ranging from 1000 to $3 \times 10^{5}$ patterns were processed. The RIP workflow (Nanao et al., 2005) was then utilized to determine heavy-atom substructures and determine phases.

\section{Phasing}

The first step in experimental phasing by HI-RIP is the determination of the 'damage substructure'. RIP substructures are generally qualitatively different from those of anomalous dispersion and isomorphous replacement, and require specialized methods (Nanao et al., 2005; de Sanctis \& Nanao, 2012). Briefly, because RIP substructures consist of many weak sites, iterative improvement of substructures and the down-weighting of the damaged (high fluence in HI-RIP) dataset are necessary. The down-weighting of the damaged dataset is carried out in a simple manner in which an initial scale is determined using conventional means, and then downweighted by the scale factor $k$. In this approach, a $k$ of 1 is the same as not applying down-weighting. In some cases, both techniques are required for the successful determination of the damage substructure, whereas in others only one of these methods is sufficient. Furthermore, it has been observed that a peak in the correlation coefficient (CC) of SHELXD (Sheldrick, 2010) substructures as a function of scale factor $(k)$ is an excellent predictor of RIP signal, which complements the conventional indicators of signal strength such as $R_{\text {isomorphous }}$ (Nanao et al., 2005). In comparing the high- and low-fluence datasets, such a relationship is observed (Fig. 2). Because the damage substructure and a reference phases set from a refined model are known, it could also be demonstrated that the $F_{\text {low fluence }}-F_{\text {high fluence }}$ map calculated with model phases by ANODE (Thorn \& Sheldrick, 2011) showed peaks, and that these peak heights (called 'RIP peak heights') were quite high (up to $25.6 \sigma$ ) over sulfur positions in methionine and cysteines, as expected from Fig. 1. The top ten strongest peaks were over the sulfur positions of residues C107, C219, M131, C154, C122, C136, M138, C119, C158 and C192 with peak heights of 25.6, 25.3, 24.9, 24.1, 28.9, 23.7, 23.2, 22.9, 22.4 and 22.2 $\sigma$, respectively. Model phases are obviously not available when trying to determine a new structure. Therefore, more practically useful measures of whether there is adequate signal are the substructures quality metrics that are produced by SHELXD. The most frequently used metric in substructure solution is the ratio of $\mathrm{CC}$ (all) to $\mathrm{CC}$ (weak). In a plot of $\mathrm{CC}($ all) against $\mathrm{CC}$ (weak), a contrast between trials with high values of $\mathrm{CC}($ all $) / \mathrm{CC}$ (weak) typically indicates that substructures are at least partially correct. Plots of $\mathrm{CC}($ all $) / \mathrm{CC}$ (weak) for the simulated data do indeed reveal a contrast in solutions (as shown in Fig. 2), with the best occurring at a $k$ of slightly less than 1.0, which is consistent with that observed in synchrotron RIP (Nanao et al., 2005). Because in this test the 'correct' substructure is known from a peak search of the RIP peak map from $A N O D E$, the resultant substructures could also be compared with this reference structure with iotbx.emma (Adams et al., 2010), which revealed that substructures were largely correct at a variety of values of $k$, with a maximum correctness at $k=0.96$ (Fig. 3). Taken together, these results 


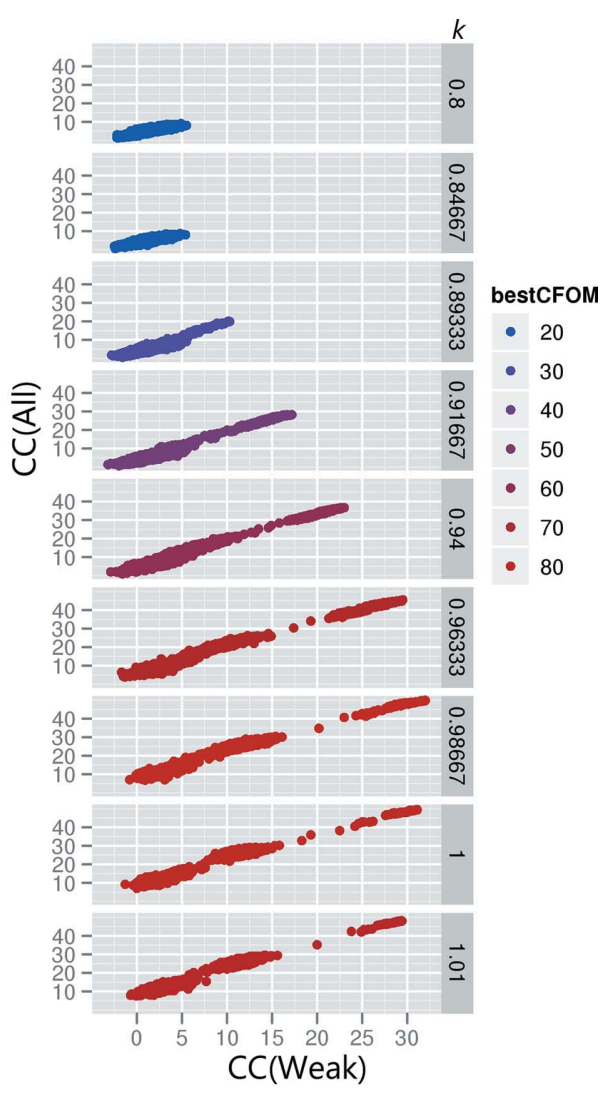

Figure 2

SHELXD correlation coefficient ratios $\left(\mathrm{CC}_{\mathrm{all}} / \mathrm{CC}_{\text {weak }}\right)$ as a function of $k$ for high fluence $=1 \times 10^{13}$ photons $\mu \mathrm{m}^{-2}$, low fluence $=1 \times 10^{11}$ photons $\mu \mathrm{m}^{-2}, 100000$ patterns.

indicate that there is adequate signal in a HI-RIP experiment to determine correct radiation damage substructures, in the same manner as in RIP.

Next, it was determined whether, together with the experimentally determined substructures, the differences between high- and low-fluence datasets were enough to determine phases. The best substructures from each $k$ were input into SHELXE (Sheldrick, 2010) for phase calculation, phase improvement and model building. Initial figure-of-merit weighted phase errors (after one round of phase improvement by SHELXE) were very good at $38^{\circ}$, and this improved and converged to $28^{\circ}$ after an additional round of substructure and phase improvement. Unlike in synchrotron X-ray and UV RIP, where the main benefit from substructure improvement is the identification of sites with 'negative' electron density in RIP difference maps caused by, for example, side chain rearrangements, these rearrangements are not anticipated on the time scale of an SFX experiment. Therefore, in this case, substructure improvement serves the purpose of identifying weaker sites in the $F_{\text {low fluence }}-F_{\text {high fluence }}$ map that were not identifiable initially by $S H E L X D$.

Having established that, in the initial testing conditions used here, standard RIP analysis could be used to both determine radiation damage substructures and to produce interpretable electron density maps, the effects of various changes to the experimental conditions were studied. Since

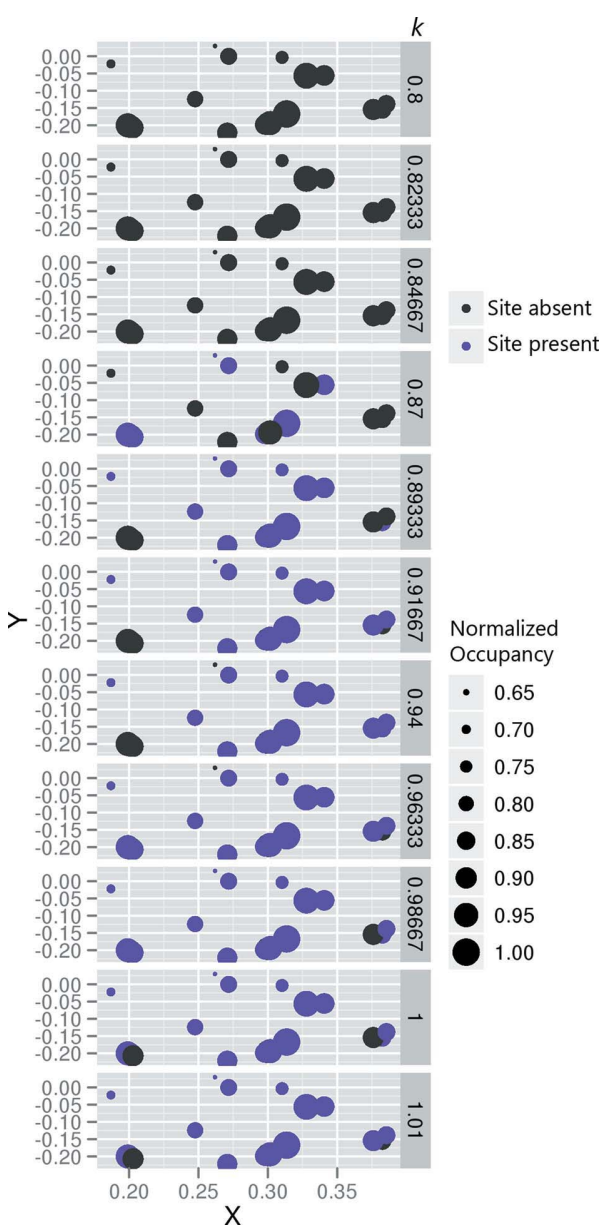

Figure 3

Correctness of SHELXD substructures. The substructure with the highest $\mathrm{CC}_{\text {all }} / \mathrm{CC}_{\text {weak }}$ was compared with a reference substructure from $A N O D E$ using phenix.emma. Purple sites indicate a correctly identified atom. Black sites indicate that a site was not identified. $X$ and $Y$ are the fractional unit-cell coordinates of the sites, as a fraction of the $X$ and $Y$ axis. The diameter of each site represents the RIP peak height, and is normalized to the most intense peak height in the difference map.

the number of patterns that can be collected during an X-ray FEL experiment is often limited due to practical reasons such as limited beam time and/or sample, and the fact that $10^{13}$ photons $\mu \mathrm{m}^{-2}$ is a current upper limit for the photon density in a single FEL pulse, the effect of both parameters on phasing were simulated. It was found that substructure solution and correct phases could be achieved with high fluences of $5 \times 10^{12}$ photons $\mu \mathrm{m}^{-2}$ even down to 20000 patterns [see, for example, the purple curve in the weighted mean phase error (wMPE) of Fig. 4], a value that is comparable with the average number of patterns required for solving SFX structures with standard methods (Boutet et al., 2012; Barends et al., 2014). With a very large number of patterns $\left(n=3 \times 10^{5}\right)$, slightly lower fluences of $1 \times 10^{12}$ photons $\mu \mathrm{m}^{-2}$ could be used as well. It is important to note that the critical parameter is not the ratio of fluences but rather the difference between fluences. This is because the effective scattering strength of sulfur does not decrease linearly with increasing fluence, but its relative change is the highest between $5 \times 10^{12}$ and 


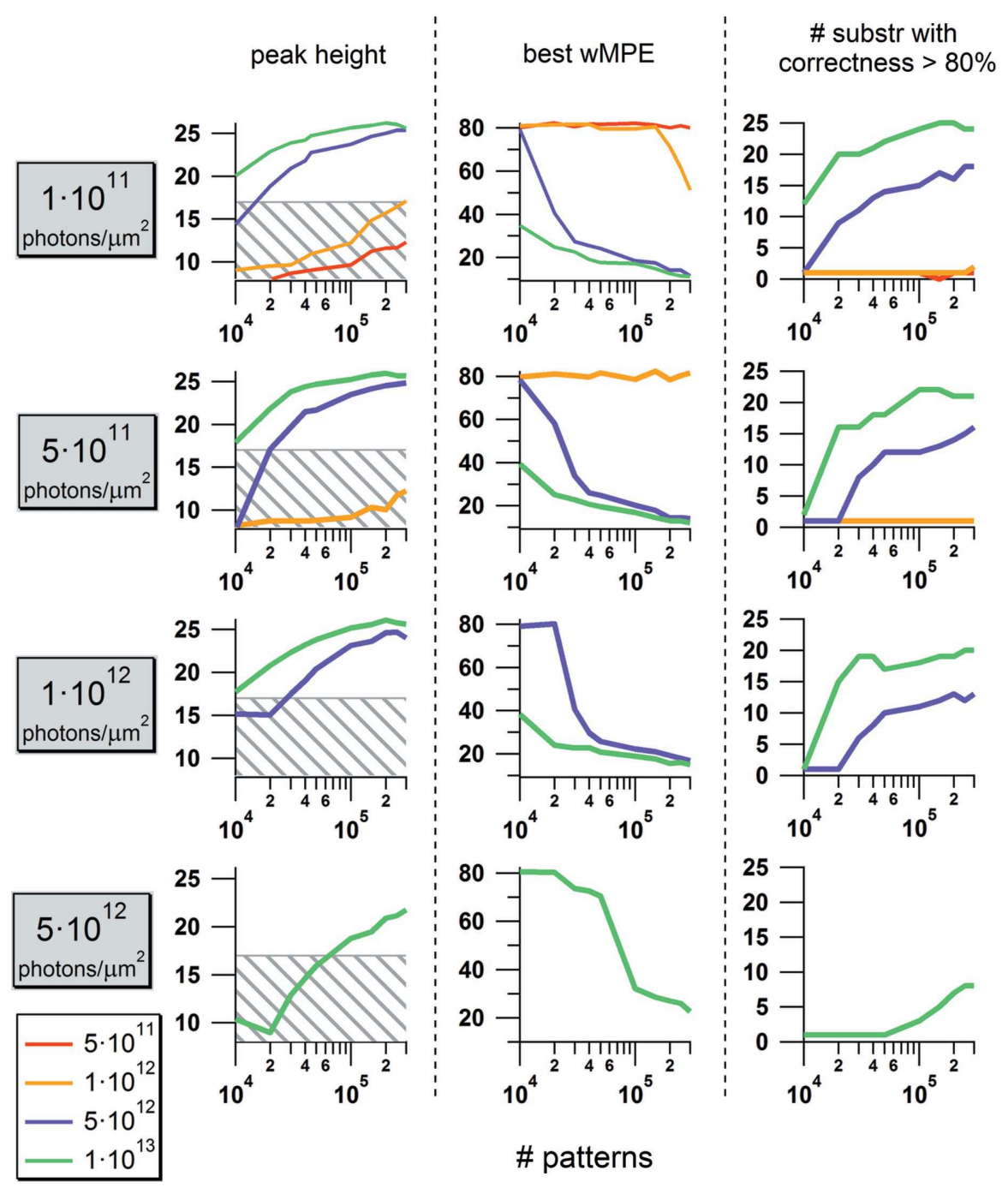

Figure 4

RIP peak height, best weighted mean phase error (wMPE), and the number of substructures having a correctness greater than $80 \%$, as a function of the number of patterns used. The corresponding low fluence is written in the grey boxes, while the high fluence is shown with different colours (see legend in the bottom-left corner).

$10^{13}$ photons $\mu \mathrm{m}^{-2}$ (see Fig. 1). In general, a larger number of patterns improves the quality of the HI-RIP solution because of improved averaging of errors in the Monte Carlo integration of intensities.

\section{Discussion and conclusions}

The success of the HI-RIP technique relies on the relative strength of the scattering factors of the heavy atoms at the two fluences used, and on the accuracy of the structure factor measurement. The former is mainly related to the specifications of the experimental facility, such as the available photon flux and bandwidth, its shot-to-shot variation and the spatial photon distribution inside the beam profile. The latter is influenced by errors introduced by the SFX technique, such as reflection partiality, inhomogeneous crystal size or quality and non-isomorphism. The Monte Carlo integration of intensities can help to average out these error sources, but a large number of observations are required. Noise was added to the simulations reported here, which yielded data with merging statistics similar to those found with experimental data, but the simulations make several assumptions that might be difficult to achieve using current FEL technology. First, it is possible that the shot-to-shot variation in intensities might contaminate the high-fluence diffraction data with data recorded at lower fluences, reducing the ionization contrast. This could be avoided by equipping the beamline with a beam diagnostic monitor after the interaction region, which could be used to sort the diffraction snapshots as a function of the photon fluence. Second, the actual beam profile is not a top-hat function but has wings of lower intensity surrounding the focus. Crystals which pass through these regions would be exposed to lower intensities than those passing through the focal spot. Currently there do not exist published beam profiles for either of the optical layouts at CXI. We have, however, used our workflow to make crude estimates of the effect of a more Gaussian beam profile, by considering high-fluence datasets created by averaging intensities from snapshots at three different photon flux densities: $10^{13}, 5 \times 10^{12}$ and $10^{12}$ photons $\mu \mathrm{m}^{-2}$. Since the lowfluence data are only affected by a negligible ionization effect, a single set at $10^{11}$ photons $\mu \mathrm{m}^{-2}$ was used. It was seen that, using 50000 patterns, phasing is still possible as long as less than $40 \%$ of the reflection intensity measurements are generated from the tail of the beam. In particular, a dataset composed of 20000 patterns at highest fluence, 20000 at medium and 10000 at low fluence showed a RIP peak height of about 21 and a best wMPE of about $20^{\circ}$ (roughly twice as large as in the pure beam case). Doubling the number of low-fluence patterns at the expense of higher fluence data reduces the RIP contrast much below $16 \sigma$, so that no phasing is possible. This indicates that the HI-RIP method can tolerate a more Gaussian beam profile. The simulation, however, does not fully model the case of a Gaussian beam of the same (or smaller) size as the crystalline sample, where the single diffraction pattern will contain both high-fluence and low-fluence scattering. This condition must be modeled with a new set of equations, by introducing different electronic responses to various X-ray fluences over the sample. Experimentally, these problems could potentially be avoided by using an X-ray beam size much larger than the crystal size. To treat the ionization 
dynamics in the present work, an independent atomic model, ignoring charge rearrangement with neighboring atoms, has been used. The molecular environment will affect all anomalous coefficients calculated within the independent atomic model. In our model, resonant absorption processes, shakeup or shakeoff processes (Persson et al., 2001), and collisional ionization (Caleman et al., 2009), which induce further ionization are also neglected. These assumptions, and no doubt others, might cause a discrepancy between simulated data and experimental data and will be incorporated into future simulations.

To conclude, SFX data at high and low photon fluence have been simulated and it has been shown that standard RIP software can be used to both determine substructures and produce interpretable electron density maps. Furthermore, it has been demonstrated that phasing can be successful using wide ranges of fluences and numbers of patterns. Future work should include an experimental demonstration of the technique, taking into account the above points and using the simulations presented here as a guide for planning.

\section{APPENDIX $A$ \\ Scattering intensity including ionization dynamics for all atomic species}

Including all types of ionization dynamics, the scattering intensity (per unit solid angle) can be evaluated by (Son et al., 2011, 2013)

$$
\begin{aligned}
\frac{\mathrm{d} I(\mathbf{Q}, \mathcal{F}, \omega)}{\mathrm{d} \Omega}= & \mathcal{F} C(\Omega) \int_{-\infty}^{\infty} \mathrm{d} t g(t) \sum_{I} P_{I}(\mathcal{F}, \omega, t) \\
& \times\left|\sum_{X} \sum_{j=1}^{N_{X}} f_{X, I_{j}^{X}}(\mathbf{Q}, \omega) \exp \left(i \mathbf{Q} \cdot \mathbf{R}_{j}^{X}\right)\right|^{2},
\end{aligned}
$$

where $g(t)$ is the normalized pulse envelope, $C(\Omega)$ is a coefficient given by the polarization of the X-ray pulse, and $P_{I}$ is the population of the global electronic configuration $I$. This global configuration index is given by $I=$ $\left(\ldots, I_{1}^{X}, I_{2}^{X}, \ldots, I_{N_{X}}^{X}, \ldots\right)$, where $X$ is one of the atom species in the sample and $I_{j}^{X}$ indicates the electronic configuration of the $j$ th atom of $X$, which is located at the position $\mathbf{R}_{j}^{X}$. Assuming that individual atoms are ionized independently, the population of configuration $I$ can be decomposed into individual populations, $P_{I}(\mathcal{F}, \omega, t)=$ $\prod_{X} \prod_{j=1}^{N_{X}} P_{X, I_{j}^{X}}(\mathcal{F}, \omega, t)$. Using this assumption, one can expand (2) and group terms for the same species, which yields equation (1). In (1), the coefficients of $a_{X}, \tilde{a}_{X}, B_{X Y}$ and $C_{X Y}$ are a function of $(\mathbf{Q}, \mathcal{F}, \omega)$, defined by equations (3),

$$
\begin{aligned}
a_{X}(\mathbf{Q}, \mathcal{F}, \omega)= & \frac{1}{\left[f_{X}^{0}(\mathbf{Q})\right]^{2}} \sum_{I_{X}}\left[\int_{-\infty}^{\infty} \mathrm{d} t g(t) P_{X, I_{X}}(\mathcal{F}, \omega, t)\right] \\
& \times\left|f_{X, I_{X}}(\mathbf{Q}, \omega)\right|^{2}
\end{aligned}
$$

$$
\begin{aligned}
\tilde{a}_{X}(\mathbf{Q}, \mathcal{F}, \omega)= & \frac{1}{\left[f_{X}^{0}(\mathbf{Q})\right]^{2}} \int_{-\infty}^{\infty} \mathrm{d} t g(t)\left|\tilde{f}_{X}(t)\right|^{2}, \\
B_{X Y}(\mathbf{Q}, \mathcal{F}, \omega)= & \frac{2}{f_{X}^{0}(\mathbf{Q}) f_{Y}^{0}(\mathbf{Q})} \\
& \times \int_{-\infty}^{\infty} \mathrm{d} t g(t)\left\{\operatorname{Re}\left[\tilde{f}_{X}(t)\right] \operatorname{Re}\left[\tilde{f}_{Y}(t)\right]\right. \\
& \left.+\operatorname{Im}\left[\tilde{f}_{X}(t)\right] \operatorname{Im}\left[\tilde{f}_{Y}(t)\right]\right\}, \\
C_{X Y}(\mathbf{Q}, \mathcal{F}, \omega)= & \frac{2}{f_{X}^{0}(\mathbf{Q}) f_{Y}^{0}(\mathbf{Q})} \\
& \times \int_{-\infty}^{\infty} \mathrm{d} t g(t)\left\{\operatorname{Re}\left[\tilde{f}_{X}(t)\right] \operatorname{Im}\left[\tilde{f}_{Y}(t)\right]\right. \\
& \left.-\operatorname{Im}\left[\tilde{f}_{X}(t)\right] \operatorname{Re}\left[\tilde{f}_{Y}(t)\right]\right\},
\end{aligned}
$$

where $I_{X}$ is the electronic configuration of the atomic species $X$ and $f_{X}^{0}(\mathbf{Q})$ indicates the normal atomic form factor of the ground configuration of $X$, which includes neither dispersion correction nor ionization. $\tilde{f}_{X}(\mathbf{Q}, \mathcal{F}, \omega, t)=\sum_{I_{X}} P_{X, I_{X}}(\mathcal{F}, \omega, t)$ $\times f_{X, I_{X}}(\mathbf{Q}, \omega)$ is the atomic form factor of $X$ at given time $t$, describing synchronized changes in the atomic form factor over all elements of the same kind. Among these coefficients, $\tilde{a}_{X}$ is corresponding to the relative effective scattering strength of $X$, as plotted in Fig. 1 .

We thank James Holton for useful discussions.

\section{References}

Adams, P. D. et al. (2010). Acta Cryst. D66, 213-221.

Barends, T. R. M. et al. (2014). Nature (London), 505, 244-247.

Boutet, S. \& Williams, G. J. (2010). New J. Phys. 12, 035024.

Boutet, S. et al. (2012). Science, 337, 362-364.

Caleman, C., Ortiz, C., Marklund, E., Bultmark, F., Gabrysch, M., Parak, F. G., Hajdu, J., Klintenberg, M. \& Tîmneanu, N. (2009). Europhys. Lett. 85, 18005.

Chapman, H. N. et al. (2011). Nature (London), 470, 73-77.

Emma, P. et al. (2010). Nat. Photon. 4, 641-647.

Evans, G., Polentarutti, M., Djinovic Carugo, K. \& Bricogne, G. (2003). Acta Cryst. D59, 1429-1434.

Hart, P. et al. (2012). Proc. SPIE, 8504, 85040C.

Hau-Riege, S. P., London, R. \& Szoke, A. (2004). Phys. Rev. E, 69, 051906.

Holton, J. M., Classen, S., Frankel, K. A. \& Tainer, J. A. (2014). FEBS J. 281, 4046-4060.

Johansson, L. C. et al. (2012). Nat. Methods, 9, 263-265.

Kirian, R. A., Wang, X., Weierstall, U., Schmidt, K. E., Spence, J. C. H., Hunter, M., Fromme, P., White, T., Chapman, H. N. \& Holton, J. (2010). Opt. Express, 18, 5713-5723.

Nanao, M. H., Sheldrick, G. M. \& Ravelli, R. B. G. (2005). Acta Cryst. D61, 1227-1237.

Owen, R. L., Rudiño-Piñera, E. \& Garman, E. F. (2006). Proc. Natl Acad. Sci. USA, 103, 4912-4917.

Persson, P., Lunell, S., Szöke, A., Ziaja, B. \& Hajdu, J. (2001). Protein Sci. 10, 2480-2484.

Quiney, H. M. \& Nugent, K. A. (2011). Nat. Phys. 7, 142-146.

Ravelli, R. B. G., Leiros, H., Pan, B., Caffrey, M. \& McSweeney, S. (2003). Structure, 11, 217-224.

Ravelli, R. B. G., Nanao, M. H., Lovering, A., White, S. \& McSweeney, S. (2005). J. Synchrotron Rad. 12, 276-284. 


\section{radiation damage}

Redecke, L. et al. (2013). Science, 339, 227-230.

Sanctis, D. de \& Nanao, M. H. (2012). Acta Cryst. D68, 1152-1162.

Sheldrick, G. M. (2010). Acta Cryst. D66, 479-485.

Son, S.-K., Chapman, H. N. \& Santra, R. (2011). Phys. Rev. Lett. 107, 218102.

Son, S.-K., Chapman, H. N. \& Santra, R. (2013). J. Phys. B 46, 164015.

Son, S.-K. \& Santra, R. (2012). XATOM: an Integrated Toolkit for $X$-ray and Atomic Physics, revision 660. DESY, Hamburg, Germany.
Son, S.-K., Young, L. \& Santra, R. (2011a). Phys. Rev. A, 83, 033402. Ten Eyck, L. F. (1977). Acta Cryst. A33, 486-492.

Thorn, A. \& Sheldrick, G. M. (2011). J. Appl. Cryst. 44, 1285-1287.

White, T. A., Barty, A., Stellato, F., Holton, J. M., Kirian, R. A., Zatsepin, N. A. \& Chapman, H. N. (2013). Acta Cryst. D69, 12311240.

White, T. A., Kirian, R. A., Martin, A. V., Aquila, A., Nass, K., Barty, A. \& Chapman, H. N. (2012). J. Appl. Cryst. 45, 335-341.

Winn, M. D. et al. (2011). Acta Cryst. D67, 235-242. 